\title{
Genome sequence and analysis of a broad-host range lytic bacteriophage that infects the Bacillus cereus group
}

Tarek F El-Arabi ${ }^{1,2}$, Mansel W Griffiths ${ }^{2,3}$, Yi-Min She ${ }^{4}$, Andre Villegas ${ }^{5}$, Erika J Lingohr ${ }^{5}$ and Andrew M Kropinski ${ }^{5,6^{*}}$

\begin{abstract}
Background: Comparatively little information is available on members of the Myoviridae infecting low $\mathrm{G}+\mathrm{C}$ content, Gram-positive host bacteria of the family Firmicutes. While numerous Bacillus phages have been isolated up till now only very few Bacillus cereus phages have been characterized in detail.

Results: Here we present data on the large, virulent, broad-host-range B. cereus phage vB_BceM_Bc431v3 (Bc431v3). Bc431v3 features a 158,618 bp dsDNA genome, encompassing 239 putative open reading frames (ORFs) and, 20 tRNA genes encoding 17 different amino acids. Since pulsed-field gel electrophoresis indicated that the genome of this phage has a mass of 155-158 kb Bc431v3 DNA appears not to contain long terminal repeats that are found in the genome of Bacillus phage SPO1.

Conclusions: Bc431v3 displays significant sequence similarity, at the protein level, to B. cereus phage BCP78, Listeria phage A511 and Enterococcus phage ØEF24C and other morphologically related phages infecting Firmicutes such as Staphylococcus phage K and Lactobacillus phage LP65. Based on these data we suggest that Bc431v3 should be included as a member of the Spounavirinae; however, because of all the diverse taxonomical information has been addressed recently, it is difficult to determine the genus. The Bc431v3 phage contains some highly unusual genes such as gp143 encoding putative tRNA ${ }^{\text {His }}$ guanylyltransferase. In addition, it carries some genes that appear to be related to the host sporulation regulators. These are: gp098, which encodes a putative segregation protein related to FstK/SpollIE DNA transporters; gp105, a putative segregation protein; gp108, RNA polymerase sigma factor F/B; and, gp109 encoding RNA polymerase sigma factor G.
\end{abstract}

Keywords: Bacillus cereus, Bacteriophage, Myoviridae, Host range, Genome, Proteome, tRNAs

\section{Background}

The genus Bacillus can be subdivided into two groups: the Bacillus subtilis group and the Bacillus cereus group [1]. Members of the latter subdivision include B. anthracis, the causative agent of the fatal human and animal disease, anthrax [2], B. cereus, B. weihenstephanensis, B. thuringiensis, B. mycoides and B. pseudomycoides. B. cereus and $B$. weihenstephanensis are implicated in foodborne illnesses as well as food spoilages. Their psychrotrophic

\footnotetext{
* Correspondence: kropinsk@queensu.ca

${ }^{5}$ Public Health Agency of Canada, Laboratory for Foodborne Zoonoses,

Guelph, ON N1G 3W4, Canada

${ }^{6}$ Department of Molecular and Cellular Biology, University of Guelph, Guelph, ON N1G 2W1, Canada

Full list of author information is available at the end of the article
}

properties enable them to cause problems in foods stored at low temperatures especially dairy products [3-6]. B. thuringiensis, B. mycoides and B. pseudomycoides have also been noted to cause food spoilage [7]. In addition, there is some evidence to indicate they potentially can cause foodborne illnesses [1,8-11].

With the exception of phages AP50, AP50-04, AP50-11, AP50-23, AP50-26, AP50-27, and Bam35 which belong to the family of Tectiviridae [12,13], all other Bacillus phages belong to the three tailed phage families: Myoviridae, Siphoviridae and Podoviridae. They range from very large viruses such as the G, to very small phages such as $\varphi 29$ and $\varphi 15$. All of the $B a$ cillus phages possess dsDNA, and some of them are quite distinct as they contain unusual bases in their DNA. In the case of phage SPO1, thymine residues are

\section{Biomed Central}


replaced with 5-(hydroxymethyl) uracil (HmUra); while in phage PBS2 DNA the thymine are totally replaced by uracil [14]. Among the myoviruses, the subfamily Spounavirinae (SPO plus "una", Latin for "one") is comprised of two genera, "SPO1-like viruses" and "Twort-like viruses". Members of Spounavirinae feature isometric heads $87-94 \mathrm{~nm}$ in diameter and tails $140-219 \mathrm{~nm}$ long [15-17]. The dsDNA molecules of this subfamily are quite large (127-142 kb) and nonpermuted with $3.1-$ $20 \mathrm{~kb}$ terminal redundancies. The genus "Spollikevirus" contains at least ten Bacillus phages, Lactobacillus phage 222a as well as Enterococcus faecalis phage ØEF24C with only the genomes of SPO1 and ØEF24C phages having been sequenced [16,18-21]. The distinct criterion of their genomes (i.e., "Spo1likevirus") is the substitution of thymine with HmUra, and the presence dUMP hydroxymethylase activity [16]. The "Twortlikevirus", on the other hand, contains a group of virulent phages of Staphylococcus including Twort, G1 and $\mathrm{K}$ phages as well as Listeria phages A511 and P100 [22-25]. As far as we know the DNA of these phages is not modified.

It is worthwhile mentioning that because of the close relatedness between members of $B$. cereus group; most of the phages isolated against $B$. anthracis were also able to infect $B$. cereus and vice-versa. The first phage reported against $B$. anthracis was phage $\mathrm{W}$, which was originally induced from a soil strain of B. cereus [26]. A lytic variant of W, phage $\gamma$, was isolated shortly after and it is now used as a standard protocol for $B$. anthracis identification by the Centers for Disease Control and Prevention and other public health laboratories in the United States [27,28].

Very few phages of $B$. cereus have been isolated and characterized in detail. Some temperate phages were characterized and utilized in the transductional analyses of $B$. cereus strains [25,29-33]. In addition, a number of lytic phages have been isolated and used in phage typing schemes for epidemiological studies [34]. It is noteworthy that phage typing could be considered as an efficient tool especially because of the close relatedness between $B$. cereus, $B$. thuringiensis and $B$. anthracis, providing a cheap, convenient and fairly accurate tool to identify such closely related species. Lytic phages have also been used in the control of $B$. cereus in mashed potatoes $[25,35,36]$.

In the present study, we isolated a lytic phage, vB_BceM_Bc431v3, that showed a strong potential to control B. cereus. Prior to its application in food preservation, we needed to characterize it and confirm that it will not affect the pathogenicity of this bacterium. Therefore, the objectives of this study were to determine the genomic sequence of phage Bc431v3, annotate its genes and appraise its structural functions in conjunction with existing phages present in the scientific literature and in the GenBank.

\section{Results}

\section{Isolation and morphology of Bc431v3}

Phage Bc431v3 was isolated from sewage and produced small $(1.8 \mathrm{~mm})$ clear plaques with turbid borders on $B$. cereus strain LJH431. This virus was negatively stained with $2 \%$ uranyl acetate and examined by transmission electron microscopy. The micrographs show that this phage has isometric heads $85.4 \pm 3 \mathrm{~nm}$ in diameter with individual capsomers visible. The virus possesses a long contractile tail $180 \pm 3 \mathrm{~nm}$ in length by $12 \pm 4 \mathrm{~nm}$ in width. The base plate has a cluster of projections and what appears to be a central tail fibre. Collectively these features indicate that this virus belongs to the family Myoviridae (Figure 1).

\section{Host range}

In a host range study, Bc431v3 was able to infect all tested B. cereus, B. anthracis, B. licheniformis and B. weihenstephanensis strains with different degrees of lysis (Table 1; Figure 2). Phage Bc431v3 also infected B. thuringiensis, B. psychrosaccharolyticus and B. megaterium, but was not able to lyse a strain of $B$. subtilis.

\section{One-step growth curve}

The growth characteristics of this virus in B. cereus strain LJH431 revealed a latent period of $85 \pm 5 \mathrm{~min}$ and a burst size of $318 \pm 5$ (Figure 3).

\section{Genome of phage Bc431v3}

The phage genome was sequenced to 19-fold coverage using 454 (pyrosequencing) technology. The genome is 158,618 bp long and possesses a G+C content of $39 \%$.

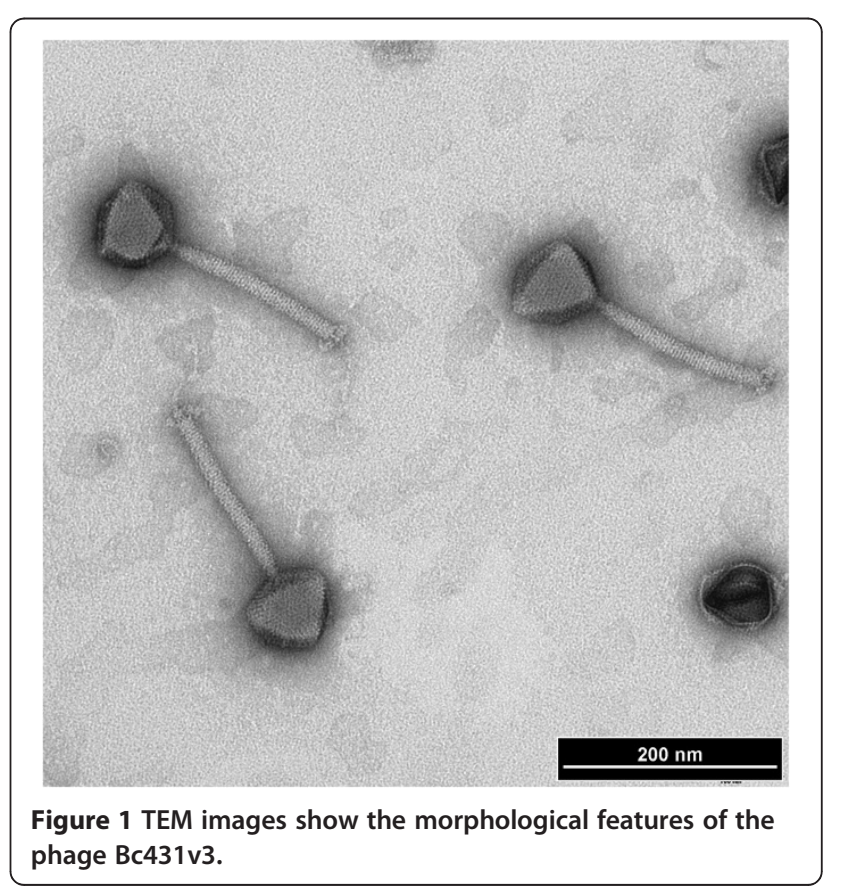


Table 1 Host range pattern of the isolated phages using Bioscreen C

\begin{tabular}{|c|c|}
\hline Host \& strains & Phage lytic pattern* \\
\hline \multicolumn{2}{|l|}{ Bacillus cereus } \\
\hline LH431 & ++ \\
\hline LH432 & ++ \\
\hline C1009 & ++ \\
\hline LH183 & ++ \\
\hline LJH181 & + \\
\hline LHH180 & ++ \\
\hline$A 2$ & ++ \\
\hline D2 & ++ \\
\hline NizoB434 & ++ \\
\hline NizoB435 & ++ \\
\hline NizoB436 & ++ \\
\hline $1230-88$ & ++ \\
\hline $75-95$ & + \\
\hline $391-98$ & ++ \\
\hline PAL-5 & ++ \\
\hline PAL-26 & + \\
\hline 17 & + \\
\hline 72 & ++ \\
\hline $67-448$ & ++ \\
\hline PAL-25 & + \\
\hline F4628/90 & ++ \\
\hline $\mathrm{F} 450 / 90$ & ++ \\
\hline PAL-18 & ++ \\
\hline \multicolumn{2}{|l|}{ Bacillus anthracis } \\
\hline$\Delta$ Sterne & ++ \\
\hline \multicolumn{2}{|c|}{ Bacillus weihenstephanensis } \\
\hline WSBC 10204 & ++ \\
\hline WSBC 10207 & ++ \\
\hline WSBC 10295 & ++ \\
\hline \multicolumn{2}{|l|}{ Bacillus thuringiensis } \\
\hline ATCC 10792 & ++ \\
\hline \multicolumn{2}{|l|}{ Bacillus subtilis } \\
\hline C1004 & - \\
\hline ATCC 6051 & - \\
\hline \multicolumn{2}{|l|}{ Bacillus licheniformis } \\
\hline C862 & + \\
\hline \multicolumn{2}{|c|}{ Bacillus psychrosaccharolyticus } \\
\hline ATCC 23296 & ++ \\
\hline \multicolumn{2}{|l|}{ Bacillus megaterium } \\
\hline ATCC 14581 & ++ \\
\hline
\end{tabular}

* Lysis pattern was measured qualitatively as (++) for full lysis, (+) for partial lysis and (-) for no lysis.

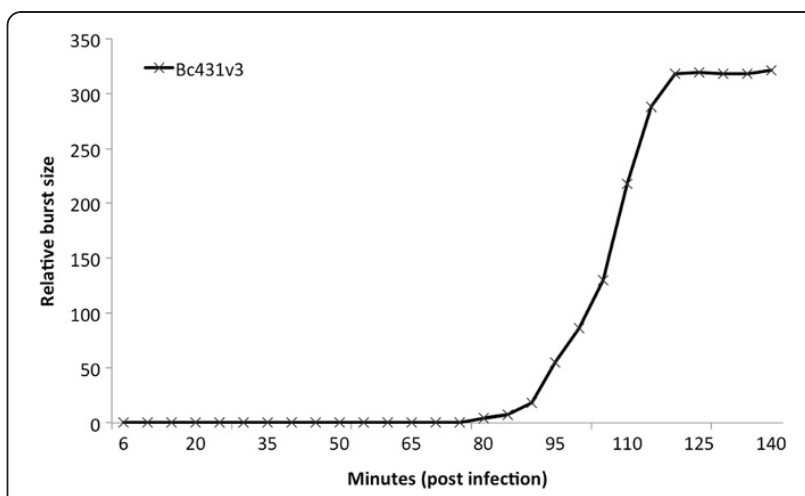

Figure 2 One-step growth curve of phage Bc431v3.

BLASTX analysis against the nonredundant NCBI database failed to reveal any frameshifts. Since pulsed-field gel electrophoresis indicated that the phage genome is a single linear DNA molecule of 155-158 kb it would suggest that this phage does not possess long terminally redundant ends such as are present on Bacillus phage SPO1.

Identification and analysis of open reading frames (ORFs) The genome was initially analyzed using AutoFACT, with the ORF calls verified using Kodon and BLAST. A total of 239 putative ORFs were identified in the genome (Figure 4; Additional file 1: Table S1). A total of 143,817 bp nucleotides (90.7\% of the genome) are involved in coding for putative proteins. Of these, 38 ORFs (15.9\%) had identified functions, while 76 ORFs (31.8\%) were identified as possessing homologs with proteins in the nonredundant NCBI database; however, their functions are undefined (conserved

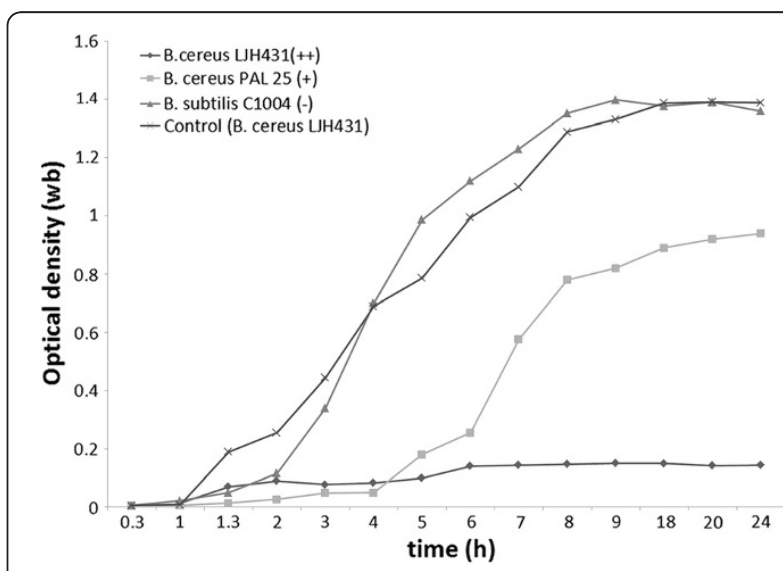

Figure 3 Representative results from Bioscreen $\mathrm{C}$ after adding phage Bc431v3 to different strains of Bacillus spp. This phage was able to completely inhibit the growth of $B$. cereus $L H 431$ (Full lysis; ++) while it only delayed the growth of B. cereus PAL 25 (Partial lysis; +). On the other hand, Bc431v3 could neither inhibit nor delay the growth of $B$. subtilis C1004. Control B. cereus $L J H 431$ refers to the growth of $B$. cereus $L J H 431$ in the absence of phage Bc431v3. 


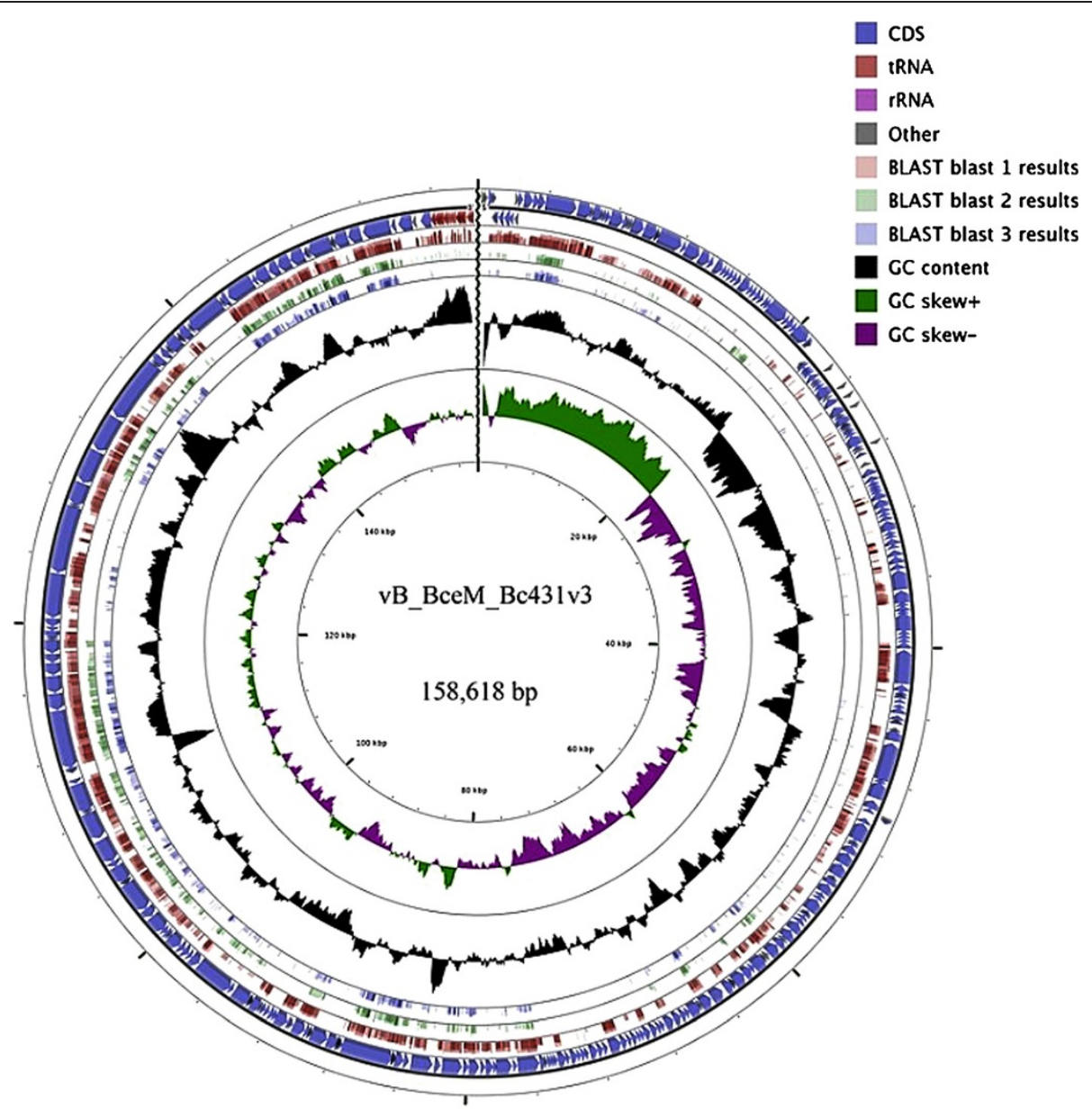

Figure 4 Genetic and physical map of phage Bc431v3 prepared using CGview (Stothard and Wishart 2005). Blast 1 shows the sequence homology between phage Bc431v3 proteins and those of $B$. cereus phage Bcp78. Blast 2 shows the homology between phage Bc341v3 and Listeria phage A511. Blast 3 shows the sequence homology between phage Bc431v3 and Enterococcus phage ØEF24C. In each of these cases TBLASTX was used to search for sequence similarity at the protein level.

hypothetical proteins). A large percentage (125 ORFs; $52.3 \%$ ) were considered to be hypothetical proteins unique to this phage. Using the homology approach introduced by Abbasifar et. al., [37] no toxins were identified among the proteins specified by this phage. Three different start codons utilized in the genome; ATG, GTG and TTG were used at frequencies $88.7 \%, 5.9 \%$ and $5.5 \%$, respectively.

The genome of this phage was exposed to DraI and SspI, EcoRI and NdeI which resulted in poor digestion, while HindIII and AccI failed to digest (data not shown). In silico restriction endonucleases digestion of phage Bc431v3 DNA revealed that the DNA possessed 200 AccI sites, $28 \mathrm{NdeI}$ sites and 23 HindIII sites, suggesting that the DNA of this virus may be modified. Two C-5 cytosine-specific DNA methyltransferases were identified in the genome encoded by genes 172 and 173, but their impact on the digestibility of the DNA is unknown.

In the following sections the properties of some of the genes will be discussed.
Nucleotide metabolism and DNA replication

Sequence-based predictions identified many genes as being involved in Bc431v3 nucleotide metabolism and DNA synthesis. The former included thymidylate synthase (gp021), ribonucleotide reductase alpha (gp185) and beta subunits (gp183), dihydrofolate reductase (gp024), deoxyuridine $5^{\prime}$-triphosphate nucleotidohydrolase (gp190), an exonuclease I homolog (gp194), and a putative exonuclease II (gp195). The proteins involved in DNA replication included a DNA polymerase (gp170), DNA primase (gp192), and two DNA helicases (gp197 and gp200).

\section{DNA packaging and morphogenesis - genomic and proteomic analysis}

Several genes encoding proteins directly involved in DNA packaging and morphogenesis were identified in Bc431v3 genome. Terminase small and large subunits homologs were determined to be the products of genes 010 and 011, respectively. Genes encoding capsid 
morphogenesis were determined to be 232, 235 and 236 for major head, prohead protease, and portal vertex proteins, respectively. Tail proteins were expressed by a set of genes, which are 224 (tail core protein), 225 (tail sheath protein), 203 and 215 (putative tail proteins), 205 and 206 (baseplate proteins); and, 204 for putative tail fiber adapter protein. It is highly unusual for the terminase genes to be separated from the capsid-tail gene complex.

A cluster of genes starting from orf203 to orf 230 carried the genes that are involved in morphogenesis, and their products were structurally similar to $B$. cereus phage Bcp78 and Listeria phage A511 [23,25]. Additionally, it was found that the product of orf218 is a putative tail protein possessing endo-beta- $\mathrm{N}$-acetylglucosaminidase activity that also contains three transmembrane domains. BLASTP homology showed that the gp218 has the motif of C-terminus similar to the putative tail lysin of the Enterococcus phage ØEF24C [19] as well as the pfam lysozyme sub-family 2. Additionally, gp218 also showed a homology with Listeria phage A511 gp97, a putative peptidase [23].

\section{Proteomic analysis}

To achieve reliable protein identification, the purified phage were digested by two different enzymes (trypsin and chymotrypsin) and analyzed by high resolution UPLC and LTQ-FT mass spectrometry in duplicates. The combined dataset from Mascot database search identified 45 phage proteins, in which the top 22 proteins were present at high scores and sequence coverages (Additional file 2: Table S2). As expected, this short list included the highly abundant structural proteins of phage Bc431v3: major capsid membrane protein (orf232; sequence coverage 50\%), tail protein (orf203; 13\%), tail sheath protein (orf225; 63\%), tail fiber protein (orf215; 14\%), tail protein possessing endo-beta- $\mathrm{N}$-acetylglucosaminidase activity (orf218; 35\%), baseplate protein (orf205; 23\%), and related structural protein (orf166; 39\%). Other phage interacting proteins and enzymes were also detected such as N-acetylmuramoyl-L alanine amidase (orf012; coverage 87\%) and prohead protease (orf235, 12\%). Prohead protease is a proteolytic enzyme essential for phage capsid morphogenesis [38], and N-acetylmuramoyl-L alanine amidase plays an important role in DNA synthesis through cleavage of amide bonds between $\mathrm{N}$-acetylmuramoyl and L-amino acids in the bacteriophage cell walls.

\section{Transcription}

We know relatively little about the regulation of transcription in large Bacillus phages. Using a combination of two techniques, visual scanning for sequences similar to SigA promoters (Sigma43; TTGACA(-35)-N $\mathrm{N}_{14}$-tgnTATAAT(-10); [39]) and MEME analysis we identified 27 putative promoters, 12 of which contained the extended -10 region (Additional file 3:
Table S3). All of these sequences possessed typical AT-rich UTR sequences, but atypically the -35 region has the consensus TTGTTGAC, not TTGACA as would be expected for promoters recognized by SigA. The majority of putative promoters were located upstream of genes involved in nucleotide metabolism (e.g. tRNA ${ }^{\text {His }}$ guanylyltransferase, dihydrofolate reductase) or DNA synthesis (DNA polymerase, primase, polymerase) which, based upon analysis of other phages, correspond to middle or delayed early genes. Because of the lack of homologs we cannot define the early genes of this phage. One of the middle genes (orf163) specifies a protein which is related to proteins defined as sigma factors for Enterococcus phage phiEF24C (YP_001504175), Staphylococcus phage K (YP_024522), Bacillus phages SP10 (BAK53012) and SPO1 (YP_002300430). In the latter virus, gp34 is known to facilitate, along with gp33 late transcription. Based upon similarity to CGTTAGA( $\left.\mathrm{N}_{17-19}\right)$ GATATT [19] and allowing for $2 \mathrm{bp}$ mismatch we identified four putative late promoters upstream of genes 056, 162, 190 and 198. Examination product of Bc431v3 orf164 using HHPred [40], failed to demonstrate homology to SPO1 gp33.

Interestingly, the $\mathrm{Bc} 431 \mathrm{v} 3$ genome, like that of temperate phage Bcp1 [28], was found to carry two other genes encoding for bacterial-type sigma factors. These are the products of genes 108 and 109, which appear to be members of the RNA polymerase sigma $F / B$ and sigma factor $\mathrm{G}$ subfamilies, respectively. MEME analysis using a variety of Bacillus, Acyclobacillus, Geobacillus and Paenibacillus sigma factors revealed that gp108 shared the following motif $\mathrm{Wx}(9) \operatorname{Ix}(2) \operatorname{Lx}(3) \operatorname{Ex}(2) \operatorname{Ix}(6) \operatorname{KDx}(2)$ QSx (2)Ax(2)LGx(5)V with SigmaF-like proteins, while gp109 shared DDxFQxGxIGLx(3)Ix(2)FDx(6)FSTYAV with related SigG proteins in the Bacillaceae.

As with Bacillus phage SPO1 [19] the genome of Bc431v3 contains a large number of rho-independent transcriptional terminators (Additional file 3: Table S3).

\section{Lysis genes}

The final stage of the phage lytic cycle is degradation of the bacterial cell wall and release of progeny phages. The lysis of the cell wall is typically induced by two phageencoded proteins, holin and endolysin [41,42]. Holin forms a hole in the cell membrane, and endolysin passes through the hole and destroys the peptidoglycan structure $[41,42]$. Two gene products were determined to be the possible lysin, an $\mathrm{N}$-acetylmuramoyl-L-alanine amidases encoded by gene 012 and/or L-alanoyl-D-glutamate peptidase encoded by gene 222 . The former contains a MurNAc-LAA [cd02696], N-acetylmuramoyl-L-alanine amidase domain while gp222 contains a COG3584 (uncharacterized protein conserved in bacteria) domain. Both share homology to defined phage lysins. As for the holin, while ten proteins possessed two transmembrane domains, and two 
three domains, none possessed the small size generally associated with holins.

\section{Codon usage and tRNAs}

The Bc431v3 genome was found to contain 20 tRNA genes for 17 amino acids (Additional file 4: Table S4). By comparing the codon usage pattern of the phage with that of its host (Bacillus cereus) we were able to see that in ten cases the phage-encoded tRNAs may significantly enhance translation of phage mRNAs (Additional file 5: Table S5). The products of two genes 143 and 177 appear to encode proteins, a tRNA ${ }^{\text {His }}$ guanylyltransferase [43] and predicted a nucleotidyltransferase, respectively, which may modify the phage or host tRNAs. As is quite common, this virus also includes a putative tRNAMet, which TFAM identified as a selenocysteinyl tRNA [44].

\section{Unusual genes}

The phage's Bc431v3 genome was found to contain several genes that have rarely been detected in other phages. In addition to sigma factors which are related to similar host proteins, we identified a putative segregation protein (gp098) related to FtsK-SpoIIIE, a DNA-binding protein (gp174) related to integration host factor (IHF), and a putative segregation protein gp105.

\section{Taxonomy}

Using BLASTN the sequence of Bc431v3 showed significant sequence similarity to the terminases of $B$. cereus phage Bcp1 [45], and B. anthracis phages PlyM19 [46] and $1102^{\varphi} 3-1$ [28]. At the protein level TBLASTX analysis (Figure 4) revealed similarity between Bc431v3 and Listeria phage A511 and Enterococcus phage ØEF24C localized to regions: $5^{\prime}$-end to $20 \mathrm{~kb}, 60-120 \mathrm{~kb}$, and $130 \mathrm{~kb}-3^{\prime}$-end. The $20-60 \mathrm{~kb}$ regions encode a wide range of hypothetical and conserved hypothetical proteins, plus the FtsK-SpoIIIE, and two sporulation-specific sigma factors, while the $120-130 \mathrm{~kb}$ region species a number of minor structural proteins. More detailed analysis using CoreGenes $[47,48]$ showed that this phage shared almost $36.4 \%$ sequence homology with Listeria phage A511 and Enterococcus phage ØEF24C while it only showed $24.3 \%$ sequence homology with Bacillus phage SPO1. In a recent reclassification of the Myoviridae, the subfamily Spounavirinae was proposed [16] with two genera the "Spollikevirus" [23] and the "Twortlikevirus." Recently a considerable number of new complete genomes of Bacillus and Staphylococcus genomes [49] have been deposited in GenBank. Rather than verifying the ICTV taxonomy proposal (2009.009a-pB.A.v3) or the paper by Klumpp et al. [18] the new data, as shown through CoreGenes proteomic analyses and phylogeny (Figure 5) strongly suggests that the Spounavirinae are far more diverse than originally realized. It would, we think be premature to assign Bc431v3 to any genus in the Spounavirinae.

\section{Discussion}

In this study we studied the biological, genomic and proteomic characteristics of Bc431v3, a strong lytic phage that is capable infecting a broad range of Bacillus species in general and members of $B$. cereus group in particular. This virus lacked repressor, site-specific integrase, virulence and antibiotic resistance determinants, which increase its potential application to the biocontrol of members of the B. cereus group.

One of the unique features of this virus is the presence of several rare or unique genes. Gene 174 encodes a putative DNA-binding protein related to bacteria integration host factor (IHF). The latter protein is nucleoid-associated and is implicated in a number of chromosomal functions including DNA compaction [45]. Bc431v3 also carries several genes directly related to host sporulation regulators, such as gene 105 encoding a putative segregation protein related to the FstK/ SpoIIIE family of DNA transporters (DNA translocase). DNA transporters are responsible for the translocation of chromosomes after the completion of genome division. In Bacillus, the DNA translocase SpoIIIE is involved in transporting miss-segregated DNA during vegetative growth $[50,51]$ and also plays an essential role in chromosome segregation during the process of sporulation [52]. Additionally, genes 108 and 109 encode sigma factor $\mathrm{F} / \mathrm{B} \sigma^{\mathrm{F} / \mathrm{B}}$ and sigma factor $\sigma^{\mathrm{G}}$, respectively. After verification of protein motifs with MEME software, we found that gp108 displays $>70 \%$ sequence similarity to Bacillus sigma factors $\mathrm{F}$ and $\mathrm{B}$ while gp109 is >80\% related to Bacillus sigma factor G. These proteins are both forespore transcription factors functioning respectively early and late in sporulation [50]. Occurrence of sporulation-related transcriptional factors on the phage Bc431v3 genome raises intriguing questions about their role in phage transcription. Schuch and Fischetti noted similar sigma factors $\mathrm{F}$ and $\mathrm{G}$ genes on the temperate phage Bcp1 genome that infects B. anthracis [30].

\section{Materials and methods}

\section{Bacterial strains and culture media}

Bacillus cereus strain LJH431 used in this study was obtained from the Canadian Research Institute for Food Safety culture collection (CRIFS, University of Guelph, Guelph, ON, Canada). Another fifteen strains of B. cereus were provided generously by T. Abee (Laboratory of Food Microbiology, Wageningen University, Netherlands). Strains A2 and D2 of B. cereus were kindly provided by A. Hudson (Christchurch Science Centre, Institute of Environmental Science and Research, New Zealand). Strains of B. weihenstephanensis were 


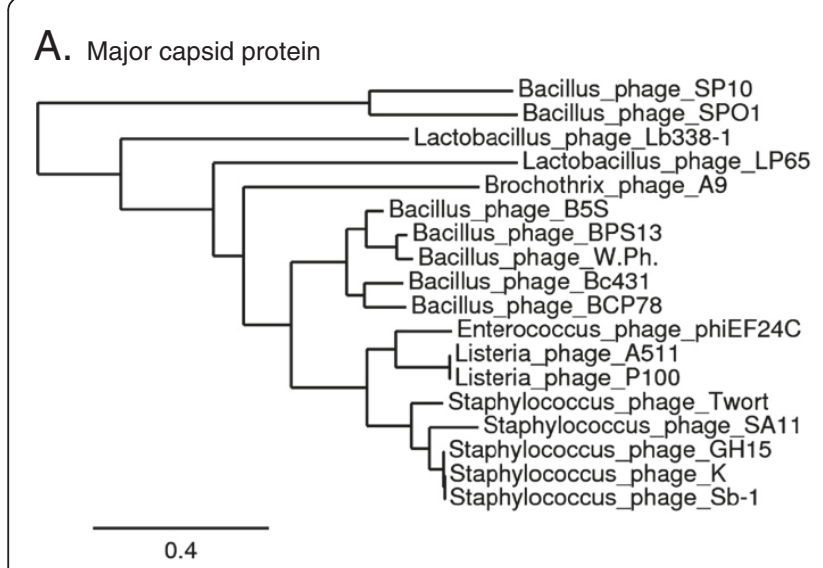

B. Tail sheath protein

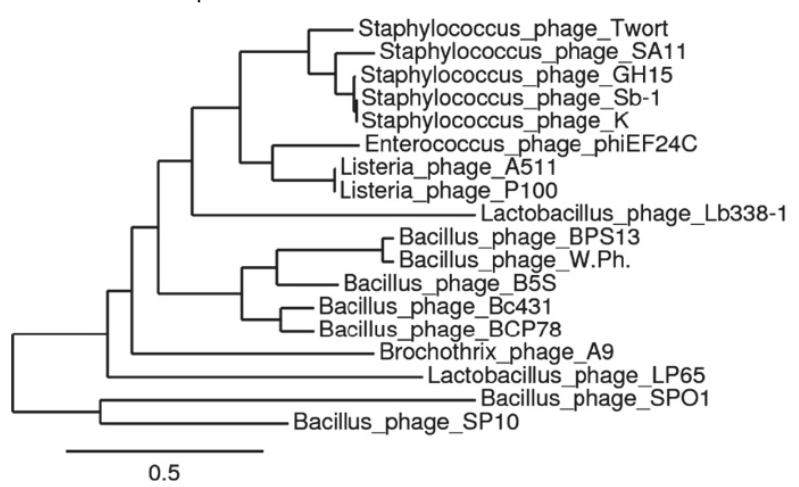

C. DNA polymerase

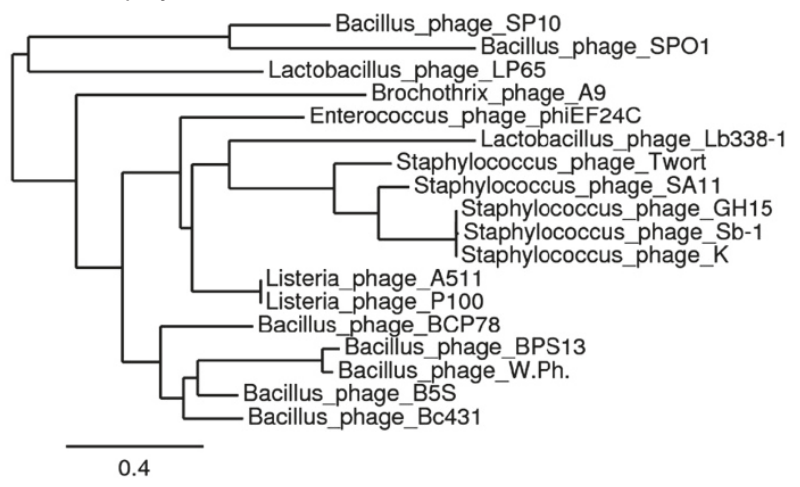

D. RecA recombinase

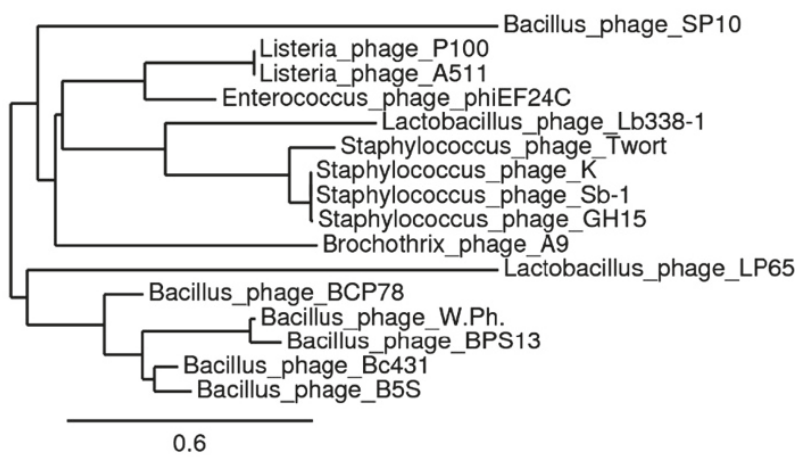

Figure 5 Phylogenetic analysis of Bc431v3 proteins using "one click" at phylogeny.fr (http://www.phylogeny.fr/version2_cgi/ simple_phylogeny.cgi): A. Major capsid protein, B. Tail sheath protein, C. DNA polymerase, and D. RecA recombinase.

kindly provided by S. Scherer (Department of Biosciences, Wissenschaftszentrum Weihenstephan, Germany). Finally B. anthracis $\Delta$ Sterne was obtained from K. Amoako (Canadian Food Inspection Agency, Ottawa, ON).

$B$. cereus strain LJH431 was grown in half-strength media of Tryptic Soy Broth (TSB; Difco Laboratories, Detroit, MI), half-strength Tryptic Soy Agar (TSA: TSB plus $1.5 \%$ agar) and half-strength Tryptic Soy Soft Agar $\left(\mathrm{TSB}+0.5 \%\right.$ agar) at $30^{\circ} \mathrm{C}$ for $24 \mathrm{~h}$.

\section{Transmission electron microscopy}

The morphology of the phage Bc431v3 was examined by transmission electron microscopy as described [53]. Briefly, $1 \mathrm{ml}$ of each phage $\left(\sim 9 \log _{10} \mathrm{PFU} \mathrm{ml}{ }^{-1}\right)$ was centrifuged at $16,000 \times \mathrm{g}$ for $1 \mathrm{~h}$ at $4^{\circ} \mathrm{C}$ (Beckman J-20 centrifuge, Beckman Coulter Inc., Mississauga, ON), pellets were washed once using $\mathrm{CM}$ buffer $\left(2.5 \mathrm{~g} / \mathrm{L} \mathrm{MgSO}_{4} .7 \mathrm{H}_{2} \mathrm{O}\right.$; $0.05 \mathrm{~g} / \mathrm{L}$ gelatin; $6 \mathrm{ml} / \mathrm{L} 1 \mathrm{M}$ Tris buffer; $0.735 \mathrm{~g} / \mathrm{L}$ $\left.\mathrm{CaCl}_{2} ; \mathrm{pH} 7.5\right)$ and then resuspended in $300 \mu \mathrm{l}$ of $\mathrm{CM}$ buffer. Five microliters of the phage suspension were applied onto 300-mesh copper grids coated with formvar and allowed to stand for $1 \mathrm{~min}$. The excess liquid was removed by filter paper and grids were stained with $2 \%$ uranyl acetate for $30 \mathrm{~s}$ then carefully blotted with filter paper to remove excess stain solution. Negatively stained phages were examined with a LEO 912AB transmission electron microscope (Energy filtered TEM [EFTEM], LEO $912 \mathrm{AB}$ model at $100 \mathrm{kv}$, Zeiss, Germany).

\section{Phage isolation and purification and, extraction of DNA}

Phage vB_BceM_Bc431v3 was isolated from sludge samples collected from a local wastewater management plant, and plaque purified. Lysates were prepared using the solid propagation method as described elsewhere [54], clarified from bacterial cell debris by centrifugation (5,400× g, $15 \mathrm{~min})$ and filtered through $0.45 \mu \mathrm{m}$ mixed cellulose ester (MCE) Fisher brand syringe filters (Fisher Scientific Company, Ottawa, ON, Canada). DNase I and RNase A (Sigma-Aldrich, Oakville, ON, Canada) were added to $10 \mu \mathrm{g} / \mathrm{ml}$ and held at $37^{\circ} \mathrm{C}$ for $30 \mathrm{~min}$. Subsequent DNA purification steps were carried on according to the handbook of QIAGEN ${ }^{\circledR}$ Lambda midi Kit (Qiagen Inc., Mississauga, ON, Canada). DNA concentration was determined by absorbance at $260 \mathrm{~nm}$ using a Nanodrop $^{\text {TM }} 1000$ spectrophotometer (Thermo Scientific, Ottawa, ON, Canada). 


\section{Determination of host specificity using Bioscreen C technology}

The host range of the phage Bc431v3 on the selected strains of Bacillus spp. was determined using the spot test technique and also by measuring the optical density (OD) in liquid medium of the tested bacterium in the presence of phage using the Bioscreen C Microbiology Plate Reader (Labsystems, Helsinki, Finland) as described elsewhere [53]. The following settings were used: wide band (wb) wavelength; $25^{\circ} \mathrm{C}$ incubation temperature; 10 min preheating time; kinetic measurement; measurement time $24 \mathrm{~h}$ at time intervals of $30 \mathrm{~min}$ with medium intensity shaking for $10 \mathrm{~s}$ before and after each measurement. Fifty microliters of each phage lysate were transferred to each of the 100 wells of the sterilized honeycomb plates of the Bioscreen $C$ reader (Fischer Scientific), and then each of the wells was inoculated with $125 \mu \mathrm{l}$ of an overnight bacterial culture at final concentrations of $3 \log _{10} \mathrm{CFU} \mathrm{ml} \mathrm{m}^{-1}$. The multiplicity of infection (M.O.I) used to determine the host range of the phage was around $10^{3}$. In this experimental design, three types of control samples were used: phage only in broth, bacteria only and sterile medium. The absorbance data were analyzed using the Bioscreen $\mathrm{C}$ data processing software version 5.26 (Labsystems) to determine the detection time (time required for each test well to increase by 0.3 OD units). Detection times (h:min) were converted to decimal values, averaged and the mean control detection time was subtracted from all test data for each isolate tested and expressed as detection time difference (DT diff.). Based on the host specificity, phages were divided into three groups: $(++)$, when phages completely inhibited the growth of the host bacterium; (+), indicating that phages delayed the growth of the host bacterium; and (-), indicating that the phage had no effect on bacterial growth.

\section{One-step growth curve}

Burst sizes and latent periods of the selected phages were determined by a one-step growth experiment as described by Anany [53]. Phages were added to its host bacterium at an MOI of around 0.1 and incubated in a water bath at $30^{\circ} \mathrm{C}$ for $5 \mathrm{~min}$. One $\mathrm{ml}$ was removed and added to $100 \mu \mathrm{l}$ of chloroform and mixed well. One hundred microliters of this mixture were added to $100 \mu \mathrm{l}$ of an overnight culture of the host bacterium and mixed with $4 \mathrm{ml}$ of overlay media and poured onto TSA agar plates to determine the degree of adsorption of the phage to bacterial cells. After an additional $30 \mathrm{~s}$ at $30^{\circ} \mathrm{C}$, $100 \mu \mathrm{l}$ were transferred to a tube containing $9.9 \mathrm{ml}$ of fresh TSB and then diluted 10 times in fresh TSB $\left(0.1 \log _{10} \mathrm{ml}^{-1}\right)$. One $\mathrm{ml}$ of the $10^{-1}$ dilution tube was additionally diluted 10 times in fresh TSB $\left(0.01 \log _{10}\right.$ $\mathrm{ml}^{-1}$ ) and all three tubes (the original plus $10^{-1}$ and $10^{-2}$ dilutions) were incubated in a water bath at $30^{\circ} \mathrm{C}$. After
$6 \mathrm{~min}$, samples were collected every $5 \mathrm{~min}$ for $3 \mathrm{~h}$ and phages were titrated in each respective sample as previously described. The relative burst size was determined according to the equation:

Relative burst size $=[($ Final titre - Initial titre $) /$ Initial titre]

The relative burst size at different times was plotted against time to determine the latent period.

\section{Determination of phage genome size using PFGE}

Phage genome size was determined by pulsed-field gel electrophoresis (PFGE) as described elsewhere [55]. Phage particles were embedded in 1\% Seakem Gold agarose (Mandel Scientific, Guelph, ON) and subjected to electrophoresis in $0.5 \mathrm{X}$ TBE buffer (5X: $20 \mathrm{ml}$ of 0.5 M EDTA [pH 8.0], $53 \mathrm{~g} / \mathrm{l}$ Tris base, and $27.5 \mathrm{~g} / \mathrm{l}$ boric acid) at $14^{\circ} \mathrm{C}$ for $18 \mathrm{~h}$, using a CHEF DR-III Mapper electrophoresis system (Bio-Rad, Mississauga, ON) with pulse times of 2.2-54.2 s, at $6 \mathrm{~V} / \mathrm{cm}$. Low range DNA marker and phage lambda DNA concatemers (New England Biolabs) were used as size standards. The gels stained with ethidium bromide and DNA bands were visualized under UV transillumination. PFGE results were analyzed using BioNumerics software (Applied Maths Inc., Austin, TX).

\section{Genome sequencing and annotation}

The sequencing of phage Bc431v3 DNA was carried out at the McGill University Genome Quebec Innovation Centre (Montreal, QC, Canada) using pyrosequencing (454 technology). AutoFACT automated annotation software [56] was initially used for genome annotation and then all open reading frames (ORFs) were confirmed using Kodon version 2.0 (Applied Maths). The individual proteins were analyzed using BLASTP against the protein databases at NCBI (http://www.ncbi.nlm.nih.gov). Protein motifs structures were identified using Pfam 24 (http://pfam.sanger.ac.uk/) [57]. In the case of the phage sigma factors a motif analysis was carried out using MEME v.4.5.0 [8]. Phage-encoded tRNA genes were identified with Aragorn [58] and tRNAscan-SE v.1.21 [59] using the default parameters. DNAMAN (Lynnon Corp., Vaudreuil-Dorion, QC, Canada) was used to determine the codon usage information of both Bc431v3 and its bacterial host Bacillus cereus, which also provided information on the GC content and presence of direct repeats in Bc431v3 genome. Putative promoters were identified by visual inspection for sequence similarity to TTGACA-N ${ }_{15-18}$-TATAAT, and by MEME analysis on the $5^{\prime}$ sequences extracted using extractUpStreamDNA (http:// lfz.corefacility.ca/extractUpStreamDNA/). Rho-independent terminators were determined by ARNold and TransTerm software $[60,61]$ and verified by examining the secondary structure of the DNA adjacent to polyT sequences using Mfold [62]. The physicochemical parameters of the gene products were determined using Molecule Weight 
and Isoelectric Point Finder (http://greengene.uml.edu/ programs/FindMW.html). Transmembrane domains were predicted using TMHMM v2.0 and Phobius or Split 4.0 [63-65]. Sequences of bacterial sigma factors and sigmafactor binding sites were identified through DBTBS database (http://dbtbs.hgc.jp) [39].

Genomic comparisons at the proteomic level were made using CoreGenes $[47,48]$. For alignments of multiple genomes and defining sequence homology percentage with related phages, progressive Mauve was used [66]. For genomic map visualization and annotation pipelines, the CGview software was used (http://wishart. biology.ualberta. ca/cgview/) [67].

\section{GenBank accession number}

The sequence of genome of this phage has been deposited with GenBank under accession number JX094431.

\section{Proteomic analysis}

Phage Bc431v3 purified through $\mathrm{CsCl}$ gradients, was reduced with $10 \mathrm{mM}$ dithiothreitol $\left(56^{\circ} \mathrm{C}, 1 \mathrm{hr}\right)$ and alkylated by $55 \mathrm{mM}$ iodoacetamide (room temperature, dark, $1 \mathrm{hr}$ ), and then dialyzed against $10 \mathrm{mM} \mathrm{NH} \mathrm{HCO}_{3}$, and dried by SpeedVac concentrator (Savant, Fisher scientific, Nepean, Ontario). Enzymatic digestions were performed on $\sim 20 \mu \mathrm{g}$ of the purified protein using either sequencing grade trypsin or chymotrypsin (100 ng, Roche Diagnostics $\mathrm{GmbH}$, Indianapolis, IN) for 4 hours. The digests were subsequently diluted by $0.2 \%$ formic acid and analyzed by online nanoAcquity ultra-performance liquid chromatography (UPLC, Waters, Milford, MA ) coupled with linear ion-trap Fourier transform ion cyclotron resonance (LTQFT ICR, Thermo Fisher, San Jose, CA) mass spectrometry. Peptides were trapped by a RP Symmetry C18 column $(180 \mu \mathrm{m}$ i.d. $\times 20 \mathrm{~mm}$ length, $5 \mu \mathrm{m})$ at $5 \mu \mathrm{l} / \mathrm{min}$, and subsequently separated on a C18 analytical column $(100 \mu \mathrm{m}$ i.d. $\times 100 \mathrm{~mm}, 1.7 \mu \mathrm{m}, \mathrm{BEH}$ 130) at $400 \mathrm{nl} / \mathrm{min}$. Peptide elution was achieved using mobile phases consisting of solvent A ( $0.1 \%$ FA) and solvent B (acetonitrile/0.1\% FA) at a linear gradient from $5 \%$ to $30 \%$, and then $85 \%$ of solvent B (65 min run). FT-MS scans were acquired with high resolution $(100,000)$ at the mass range of $\mathrm{m} / \mathrm{z} 300$ to 2000 , and low resolution MS/MS measurements in linear iontrap mode were obtained by data-dependent scans of the top eight most intense precursor ions at multiply charged states of $2+, 3+$, and $4+$. Dynamic exclusion was enabled for a period of $180 \mathrm{~S}$.

Protein identification was performed using an in-house Mascot Server (version 2.3.0, Matrix Science, London, UK), and the raw data were searched against the Bc431v3 protein database. The parameter settings allowed specific trypsin digestion for maximum 2 missed cleavage sites, and non-specific digestion of chymotrypsin. Cystein carbamidomethylation was designated as a fixed modification of peptides, and deamidation of asparagine and glutamine, methionine oxidation, pyro-Glu of Gln conversion at the $\mathrm{N}$-terminus were considered as variable modifications. Mass tolerances were set up to $10 \mathrm{ppm}$ for the FT MS ions and $1 \mathrm{Da}$ for ion trap MS/MS fragment ions. Peptide assignments were filtered by an ion score cut off of 20 , and the significance threshold was adjusted to 0.001 to achieve a false discovery rate (FDR) of less than 3\% [38].

\section{Additional files}

\section{Additional file 1: Table S1. General features of putative ORFs of Bc431v3 and homology to proteins in the database. In addition, protein motifs including transmembrane domains are included.}

Additional file 2: Table S2. Protein identification of phage Bc431v3 by UPLC LTQ-FT MS/MS analyses.

Additional file 3: Table S3. Potential promoters and rho-independent terminators in vB_BceM_Bc431v3 with WebLogo of the consensus.

Additional file 4: Table S4. tRNAs discovered in the sequence of Bc341v3 using tRNAScan-SE.

Additional file 5: Table S5. Comparison of codon usage in Bc431v3 genes with that of Bacillus cereus Q1. Those codons which are $50 \%$ more common in the phage are coloured orange.

\section{Abbreviations}

BLAST: Basic Local Alignment Search Tool; dsDNA: Double-stranded deoxyribonucleic acid; EDTA: Ethylenediaminetetraacetic acid; HmUra: 5Hydroxymethyluracil; MOI: Multiplicity of infection (ratio of phage to bacteria); NCBI: National Center for Biotechnology Information; ORFs: Open reading frames; PFGE: Pulsed-field gel electrophoresis; PFU: Plaque forming unit; TBE: Tris borate EDTA; TSB: Tryptic Soy Broth; TMHMM: TransMembrane prediction using Hidden Markov Models; PEG: Polyethylene Glycol.

\section{Competing interests}

The authors have no competing interests to disclose.

\section{Authors' contributions}

The bulk of the research and writing was conducted by TFEA, under the supervision of MWG. YMS carried out the proteomic studies. The initial annotation was performed by $\mathrm{AV}$, and then extended by AMK and TFEA. EJL carried out the PFGE analyses. All authors read and approved the final manuscript.

\section{Authors' information}

TFEA is currently a post-doctoral fellow in Canadian Research Institute for Food Safety, Food Science Department, University of Guelph, Ontario, Canada.

\section{Acknowledgements}

During this research A.M.K. was supported by a Discovery Grant from the Natural Sciences and Engineering Research Council of Canada. The funding body did not have any role in the design of the experiments, in the collection, analysis, and interpretation of data; in the writing of the manuscript; or in the decision to submit the manuscript for publication.

\section{Author details}

${ }^{1}$ Department of Agricultural Microbiology, Faculty of Agriculture, Ain Shams University, Cairo, Egypt. ${ }^{2}$ Canadian Research Institute for Food Safety, University of Guelph, Guelph, ON N1G 2W1, Canada. ${ }^{3}$ Department of Food Science, University of Guelph, Guelph, ON N1G 2W1, Canada. ${ }^{4}$ Centre for Vaccine Evaluation, Biologics and Genetic Therapies Directorate, Health Canada, Ottawa, ON K1A OK9, Canada. ${ }^{5}$ Public Health Agency of Canada, Laboratory for Foodborne Zoonoses, Guelph, ON N1G 3W4, Canada. ${ }^{6}$ Department of Molecular and Cellular Biology, University of Guelph, Guelph, ON N1G 2W1, Canada. 
Received: 13 August 2012 Accepted: 8 January 2013

Published: 7 February 2013

\section{References}

1. Griffiths MW: Bacillus cereus and Other Bacillus spp. In Pathogens and Toxins in Foods: Challenges and Interventions. Edited by Juneja VK, Sofos JN. Washignton, DC: ASM Press; 2010.

2. Garber KB: Anthrax (Bacillus anthracis). KGaA: Molecular Biology of. WileyVCH Verlag GmbH \& Co; 2006.

3. Bandara N, Jo J, Ryu S, Kim KP: Bacteriophages BCP1-1 and BCP8-2 require divalent cations for efficient control of Bacillus cereus in fermented foods. Food Microbiol 2012, 31(1):9-16.

4. Bottone EJ: Bacillus cereus, a volatile human pathogen. Clin Microbiol Rev 2010, 23:382-398.

5. Shin H, Bandara N, Shin E, Ryu S, Kim KP: Prevalence of Bacillus cereus bacteriophages in fermented foods and characterization of phage JBP901. Res Microbiol 2011, 162(8):791-797.

6. Stenfors L, Mayr R, Scherer S, Granum P: Pathogenic potential of fifty Bacillus weihenstephanensis strains. FEMS Microbiol Lett 2002, 215:47-51.

7. Barfod K, Poulsen S, Hammer M, Larsen S: Sub-chronic lung inflammation after airway exposures to Bacillus thuringiensis biopesticides in mice. BMC Microbiol 2010, 10:233.

8. Ankolekar C, Rahmati T, Labbé RG: Detection of toxigenic Bacillus cereus and Bacillus thuringiensis spores in U.S. rice. Int. J. Food Microbio/ 2009, 128:460-466.

9. Mclntyre L, Bernard K, Beniac D, Isaac-Renton JL, Naseby DC: Identification of Bacillus cereus group species associated with food poisoning outbreaks in British Columbia. Canada Appl Environ Microbiol 2008, 74:7451-7453.

10. Zhou G, Liu H, He J, Yuan Y, Yuan Z: The occurrence of Bacillus cereus, B. thuringiensis and B. mycoides in Chinese pasteurized full fat milk. Int J Food Microbiol 2008, 121:195-200.

11. Zhou G, Zheng D, Dou L, Cai Q, Yuan Z: Occurrence of psychrotolerant Bacillus cereus group strains in ice creams. Int J Food Microbiol 2010, 137:143-146.

12. Ackermann HW, Abedon ST; 2000. http://www.mansfield.ohio-state.edu/ s sabedon/names/2000/.

13. Ackermann HW, Azizbekyan RR, Emadi Konjin HP, Lecadet MM, Seldin L, Yu MX: New Bacillus bacteriophage species. Arch Virol 1994, 135:333-344.

14. Ackermann HW: Phage Classification and Characterization. Methods Mol Biol 2009, 501:127-140

15. Allan BJ, Davies P, Carstens EB, Kropinski AM: Characterization of the genome of Pseudomonas aeruginosa bacteriophage $\varphi$ PLS27 with particular reference to the ends of the DNA. J Virol 1989, 63:1587-1594.

16. Lavigne R, Darius PI, Summer E, Seto D, Mahadevan P, Nilsson A Ackermann $\mathrm{H}$, Kropinski A: Classification of Myoviridae bacteriophages using protein sequence similarity. BMC Microbiol 2009, 9:224.

17. Perkus ME, Shub DA: Mapping the genes in the terminal redundancy of bacteriophage SPO1 with restriction endonucleases. J Virol 1985, 56:40-48.

18. Klumpp J, Lavigne R, Loessner M, Ackermann HW: The SPO1-related bacteriophages. Arch Virol 2010, 155:1547-1561.

19. Stewart CR, Casjens SR, Cresawn SG, Houtz JM, Smith AL, Ford ME, Peebles CL, Hatfull GF, Hendrix RW, Huang WM, Pedulla ML: The Genome of Bacillus subtilis bacteriophage SPO1. J Mol Biol 2009, 388:48-70.

20. Uchiyama J, Rashel M, Maeda Y, Takemura I, Sugihara S, Akechi K, Muraoka A, Wakiguchi H, Matsuzaki S: Isolation and characterization of a novel Enterococcus faecalis bacteriophage $\varphi \mathrm{EF} 24 \mathrm{C}$ as a therapeutic candidate. FEMS Microbiol Lett 2008, 278:200-206.

21. Uchiyama J, Rashel M, Takemura I, Wakiguchi H, Matsuzaki S: In silico and in vivo evaluation of bacteriophage $\varphi \mathrm{EF} 24 \mathrm{C}$, a candidate for treatment of Enterococcus faecalis infections. Appl Environ Microbio/ 2008, 74:4149-4163.

22. Carlton RM, Noordman WH, Biswas B, de Meester ED, Loessner MJ: Bacteriophage P100 for control of Listeria monocytogenes in foods: Genome sequence, bioinformatic analyses, oral toxicity study, and application. Regul Toxicol Pharmacol 2005, 43:301-312.

23. Klumpp J, Dorscht J, Lurz R, Bielmann R, Wieland M, Zimmer M, Calendar R, Loessner MJ: The terminally redundant, nonpermuted genome of Listeria bacteriophage A511: a model for the SPO1-like myoviruses of Grampositive bacteria. J Bacteriol 2008, 190:5753-5765.

24. Kwan T, Liu J, DuBow M, Gros P, Pelletier J: The complete genomes and proteomes of 27 Staphylococcus aureus bacteriophages. Proc Natl Acad Sci USA 2005, 102:5174-5179.
25. Lee J, Shin H, Son B, Ryu S: Complete genome sequence of Bacillus cereus bacteriophage BCP78. J Virol 2012, 86:637-638.

26. Ruhfel RE, Robillard NJ, Thorne CB: Interspecies transduction of plasmids among Bacillus anthracis, B. cereus, and B. thuringiensis. J Bacterio/ 1984, 157:708-711.

27. Mccloy EW: Lysogenicity and immunity to Bacillus phage. W J Gen Microbiol 1958, 18:198-220

28. Schuch R, Pelzek AJ, Kan S, Fischetti VA: Prevalence of Bacillus anthracislike organisms and bacteriophages in the intestinal tract of the earthworm Eisenia fetida. Appl Environ Microbiol 2010, 76:2286-2294.

29. Kong M, Kim M, Ryu S: Complete genome sequence of Bacillus cereus bacteriophage PBC1. J Virol 2012, 86(11):6379-6380.

30. Schuch R, Fischetti VA: The secret life of the anthrax agent Bacillus anthracis: bacteriophage-mediated ecological adaptations. PLOS One 2009, 4:e6532.

31. Thorne CB: Transduction in Bacillus cereus and Bacillus anthracis. Microbiol Mol Biol Rev 1968, 32:358-361.

32. Thorne CB, Holt SC: Cold lability of Bacillus cereus bacteriophage CP-51. $J$ Virol 1974, 14:1008-1012

33. Yelton DB, Thorne CB: Transduction in Bacillus cereus by each of two bacteriophages. J Bacteriol 1970, 102:573-579.

34. Ahmed R, Sankar-Mistry P, Jackson S, Ackermann HW, Kasatiya SS: Bacillus cereus phage typing as an epidemiological tool in outbreaks of food poisoning. J Clin Microbiol 1995, 33:636-640.

35. Lee W, Billington C, Hudson JA, Heinemann JA: Isolation and characterization of phages infecting Bacillus cereus. Microbiol: Lett. Appl; 2011.

36. Loessner MJ, Maier SK, Daubek-Puza H, Wendlinger G, Scherer S: Three Bacillus cereus bacteriophage endolysins are unrelated but reveal high homology to cell wall hydrolases from different bacilli. J Bacterio/ 1997, 179:2845-2851.

37. Abbasifar RAM, Kropinski PM, Sabour HWA, Lingohr EJ, Griffiths MW: Complete Genome Sequence of Cronobacter sakazakii Bacteriophage vB_CsaM_GAP161.J Virol 2012, 86:13806-13807.

38. Liu J, Mushegian A: Displacements of prohead protease genes in the late operons of double-stranded-DNA bacteriophages. J Bacterio/ 2004 186:4369-4375.

39. Ishii T, Yoshida K, Terai G, Fujita Y, Nakai K: DBTBS: a database of Bacillus subtilis promoters and transcription factors. Nucl Acids Res 2001, 29:278-280.

40. Söding J, Biegert A, Lupas AN: The HHpred interactive server for protein homology detection and structure prediction. Nucl Acids Res, 33:W244-W248

41. Hanlon GW: Bacteriophages: an appraisal of their role in the treatment of bacterial infections. Int J Antimicrob Agents 2007, 30:118-128.

42. Kutter E, Sulakvelidze A: Bacteriophages: biology and applications. Boca Raton, FL: CRC Press; 2005.

43. Hyde SJ, Eckenroth BE, Smith BA, Eberley WA, Heintz NH, Jackman JE, Doublié S: tRNAHis guanylyltransferase (THG1), a unique $3^{\prime}-5^{\prime}$ nucleotidyl transferase, shares unexpected structural homology with canonical $5^{\prime}-3^{\prime}$ DNA polymerases. Proc Natl Acad Sci USA 2010, 107:20305-20310.

44. Tåquist $H$, Cui $Y$, Ardell DH: TFAM 1.0: an online tRNA function classifier. Nucl. Acids Res 2007, 35:W350-W353.

45. Sarkar T, Petrov AS, Vitko JR, Santai CT, Harvey SC, Mukerji I, Hud NV: Integration host factor (IHF) dictates the structure of polyamine-DNA condensates: implications for the role of IHF in the compaction of bacterial Chromatin. Biochemistry 2009, 48:667-675.

46. Schmitz JE, Schuch R, Fischetti VA: Identifying active phage lysins through functional viral metagenomics. App/ Environ Microbiol 2010, 76:7181-7187.

47. Kropinski AM, Borodovsky M, Carver TJ, Cerdeño-Tárraga AM, Darling A, Lomsadze A, Mahadevan P, Stothard P, Seto D, Domselaar G, Wishart DS: In silico identification of genes in bacteriophage DNA. Methods Mol Biol 2009, 502:57-89.

48. Zafar N, Mazumder R, Seto D: CoreGenes: A computational tool for identifying and cataloging "core" genes in a set of small genomes. BMC Bioinforma 2002, 3:12.

49. Łobocka M, Hejnowicz MS, Dąbrowski K, Gozdek A, Kosakowski J, Witkowska M Ulatowska MI, Weber-Dąbrowska B, Kwiatek M, Parasion S, Gawor J, Kosowska H, Głowacka A: Genomics of staphylococcal Twort-like phages-potential therapeutics of the post-antibiotic era. Adv Virus Res 2012, 83:143-216.

50. Camp AH, Losick R: A feeding tube model for activation of a cell-specific transcription factor during sporulation in Bacillus subtilis. Genes Dev 2009, 23:1014-1024 
51. Sharpe ME, Errington J: Postseptational chromosome partitioning in bacteria. Proc Natl Acad Sci USA 1995, 92:8630-8634

52. Wu L, Errington J: Use of asymmetric cell division and spolllE mutants to probe chromosome orientation and organization in Bacillus subtilis. Molec Microbiol 1998, 27:777-786.

53. Anany HE: Biocontrol of FoodBorne Bacterial Pathogens Using Immobilized Bacteriophages. Ph.D. University of Guelph: University of Guelph Libraries; 2010

54. Abedon S, Hyman P, Thomas C: Experimental examination of bacteriophage latent-period evolution as a response to bacterial availability. Appl Environ Microbiol 2003, 69:7499-7506.

55. Lingohr E, Frost S, Johnson RP: Determination of bacteriophage genome size by pulsed-field gel electrophoresis. Methods Mol Biol 2009, 502:19-25.

56. Koski L, Gray M, Lang BF, Burger G: AutoFACT: An automatic functional annotation and classification tool. BMC Bioinforma 2005, 6:151.

57. Finn RD, Mistry J, Tate J, Coggill P, Heger A, Pollington JE, Gavin OL, Gunasekaran P, Ceric G, Forslund K, Holm L, Sonnhammer ELL, Eddy SR, Bateman A: The Pfam protein families database. Nucl Acids Res 2010, 38:D211-D222.

58. Laslett $D$, Canback B: ARAGORN, a program to detect tRNA genes and tmRNA genes in nucleotide sequences. Nucl Acids Res 2004, 32:11-16.

59. Lowe TM, Eddy SR: tRNAscan-SE: a program for improved detection of transfer RNA genes in genomic sequence. Nucl Acids Res 1997, 25:955-964.

60. Ermolaeva MD, Khalak HG, White O, Smith HO, Salzberg SL: Prediction of transcription terminators in bacterial genomes. J Mol Biol 2000, 301:27-33.

61. Gautheret D, Lambert A: Direct RNA motif definition and identification from multiple sequence alignments using secondary structure profiles. J Mol Biol 2001, 313:1003-1011.

62. Zuker M: Mfold web server for nucleic acid folding and hybridization prediction. Nucl Acids Res 2003, 31:3406-3415.

63. Juretić $\mathrm{D}$, Zoranić L, Zucić D: Basic charge clusters and predictions of membrane protein topology. J Chem Inf Comput Sci 2002, 42:620-632.

64. Käll L, Krogh A, Sonnhammer ELL: Advantages of combined transmembrane topology and signal peptide prediction-the Phobius web server. Nucl Acids 2007, 35:429-432.

65. Krogh A, Larsson B, von Heijne G, Sonnhammer ELL: Predicting transmembrane protein topology with a hidden markov model: application to complete genomes. J Mol Biol 2001, 305:567-580.

66. Darling AE, Mau B, Perna NT: progressiveMauve: multiple genome alignment with gene gain, loss and rearrangement. PLoS One 2010, 5:e11147.

67. Stothard $\mathrm{P}$, Wishart DS: Circular genome visualization and exploration using CGView. Bioinformatics 2005, 21:537-539.

doi:10.1186/1743-422X-10-48

Cite this article as: El-Arabi et al:: Genome sequence and analysis of a broad-host range lytic bacteriophage that infects the Bacillus cereus group. Virology Journal 2013 10:48.

\section{Submit your next manuscript to BioMed Central and take full advantage of:}

- Convenient online submission

- Thorough peer review

- No space constraints or color figure charges

- Immediate publication on acceptance

- Inclusion in PubMed, CAS, Scopus and Google Scholar

- Research which is freely available for redistribution
C Biomed Central 